$\xi=-1$

\title{
A prediction model for peer attachment in KOREAN female adolescents using back propagation neural network
}

\author{
Haewon Byeon \\ Department of Speech Language Pathology, College of Health Science,Honam University, 417, Eodeung-daero, Gwangju, Republic of \\ Korea \\ *Corresponding author E-mail: bhwpuma@naver.com
}

\begin{abstract}
Background/Objectives: This study used data mining technique to explore the potential factors affecting the peer attachment of South Korean female students.

Methods/Statistical analysis: This study analyzed 2009 9th grade female students, who attended Panel Study on Korean Children in 2016. Peer attachment was defined as a dependent variable. The explanatory variables included gender, academic achievement satisfaction, subjective household economy level, parent-child dialogue frequency, subjective health status, depression symptom, self-esteem, subjective life satisfaction, and mobile phone dependence. The predictors of peer attachment were analyzed by using back propagation neural network (BPN).

Findings: Analysis results showed that depression, self-esteem, dialogue level between parent and child regarding school life, subjective health condition were highly related to the peer attachment of female students.

Improvements/Applications: It is required to develop a customized educational program to form a successful social relationship between adolescent female students.
\end{abstract}

Keywords: Datamining; Back Propagation Neural Network, Peer Attachment; Risk Factors; Female Students.

\section{Introduction}

People experience rapid physical, psychological, and social development during the adolescence period. Hormonal changes in this period lead to secondary sexual characteristics and people frequently experience anxiety and impulse ${ }^{1}$. Particularly, when students begin to go to school, their temporal and spatial scales expand to school in addition to the home. They improve their academic achievement and they also form identity while spending time with peers. Furthermore, they develop personality and social competence during the period [2]. Therefore, the stable attachment with peers during the adolescent period is important for successful learning and growth.

Peer attachment is an emotional bond formed between horizontal relation and it indicates the trust and intimacy between peers or a positive relationship allowing a person to exchange feelings and thoughts [2]. Adolescents learn intimacy and sociality through interactions with peers and form interpersonal relationships 3 . It is necessary to pay attention to peer attachment to enhance the peer relation because they form a relationship with other peers based on the attachment with close peers.

Previous study reported that children with the higher level of peer attachment were more likely to adapt to school life and felt competence and satisfaction in interpersonal relationships [4]. On the other hand, children with the lower level of peer attachment had a higher probability to experience emotional problems and school maladjustment 5. Consequently, it was expected that we could reduce the emotional problems including depression by identifying factors influencing peer attachment and predicting a group with a high risk of peer attachment instability to manage them in advance.

Numerous previous studies reported that peer attachment is affected by various factors including personal variables such as selfesteem and family variables such as parent-child relationship and sibling relationship [6-7]. For example, when a parent showed a positive parenting behavior, an adolescent child felt comfortable with having a close relationship with others7. Particularly, many studies reported that female students showed a higher level of peer attachment and higher desire to form relationships with others than male students8, 9. Consequently, it is urgent to identify the high-risk female student group with peer attachment instability.

However, the majority of the previous studies dealt only a portion of individual variables that could affect the peer attachment of adolescents or they tended to define and analyze peer attachment as an independent variable rather than a dependent variable2, 10. Moreover, although gender is known to affect the level of peer attachment, there are only a few studies evaluating the instability risk factor of female students' peer attachment.

Artificial neural network is widely used as a method of developing a prediction model in recent years. Particularly, back propagation neural network (BPN) has many advantages including high prediction accuracy, fast learning speed, and excellent classification ability 11 .

This study developed a peer attachment prediction model based on the BPN algorithm for 9th-grade female students in South Korea. Chapter 2 will define the study subjects and measurement variables. Chapter 3 will explain the BPN algorithm and the procedure of model development. Chapter 4 will describe the results of the peer attachment prediction model based on the BPN algorithm. Chapter 5 presents conclusion and direction for future studies. 


\section{Materials}

\subsection{Subject}

This study used the raw data of 'Panel Study on Korean Children in 2016', which was conducted by National Youth Policy Institute under the auspices of Ministry of Health and Welfare and Headquarters for Community Children's Center [12]. This study targeted 209 female students in 9th grade among all respondents.

\subsection{Measurement and definition of variables}

Dependent variables were defined by the presence of peer attachment instability. Peer attachment was measured by using 'The Inventory of Parent and Peer Attachment (IPPA)' [13], which is composed of 9 items. A higher total IPPA score is interpreted as higher peer attachment establishment. This study defined the bottom $25 \%$ of the IPPA scores as a peer attachment instability highrisk group.

Explanatory variables included gender, academic achievement satisfaction (i.e., satisfied or dissatisfied), subjective household economy level (i.e., rich, moderate, or poor), the frequency of parent-child dialogue (i.e., less than 2 times per week, 3-4 times per week, or more than 5 times per week), subjective health status (i.e., poor, normal, or healthy), depression symptoms, self-esteem, subjective life satisfaction, and mobile phone dependency.

\section{Methods}

\subsection{Exploring potential factors of peer attachment in- stability high-risk group}

The general characteristics of the peer attachment instability highrisk group were presented by using descript statistics (e.g., mean and percentage). Moreover, the difference between groups by peer attachment level was analyzed by using Chi-square test.

\subsection{Back propagation neural network}

Artificial neural network is a type of data mining algorithms that mimics the information process of the human brain. It is a nonlinear model exploring the hidden pattern of data by going through learning processes repetitively. The structure of the artificial neural network is composed of input layers, which consist of nodes corresponding to input variables, and hidden layers, which consist of hidden nodes [14]. Representative algorithms of artificial neural networks are multi-layer neural network and BPN.

BPN is composed of multiple layers (Figure. 1) and it is one of multilayer perceptron models [11]. The learning method is supervised learning and it is a process to find an appropriate weight which shall be applied to input data for acquiring target data. The output data of an artificial neural network has an error and the weight of output layer is updated in proportion to the error. Afterward, the weight of a hidden layer is updated. The out of a hidden layer in the BNP algorithm is shown equation (1).

$$
t_{j}=\phi\left(r_{j}\right)=\phi\left(\sum W_{i j} x_{i}\right)
$$

Wij is the coupling strength between an input layer and a hidden layer. $x i$ is input data and $t j$ is the output of a hidden layer delivered by the output neuron. The combine function of the output layer is weighted according to the access $\mathrm{Y}(\mathrm{k})$ for given actual output $\mathrm{y}(\mathrm{k})$ (see equation 2$)$

$$
y^{(k)} \approx Y^{(k)}=\sum_{j=1}^{n} c_{j}^{(k)} t_{j}=\sum c_{j}^{(k)} \phi \sum W_{i j} x_{i}
$$

In the analysis of artificial neural network analysis, the major predictor variables were presented by comparing the normalized importance, which uniformed the unit of the relative importance of inputs and that of the input variables.

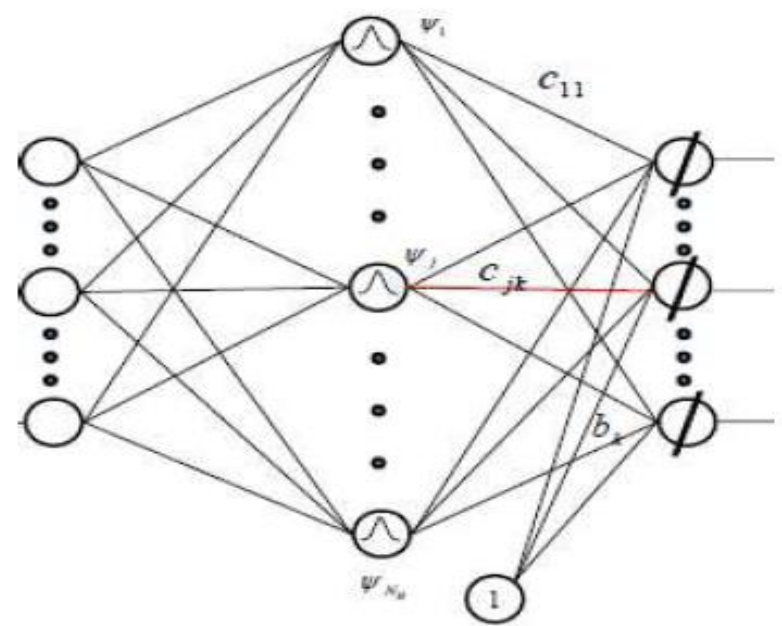

Fig. 1: Structure of the Back Propagation Neural Network.

\section{Results}

\subsection{Exploring potential factors of peer attachment in- stability high-risk group}

The general characteristics of the subjects according to the presence of peer attachment are presented in Table 1. Among 209 subjects, 48 subjects $(23.0 \%)$ were classified as the peer attachment instability high-risk group. Cross-validation analysis showed that the adolescents in the peer attachment instability high-risk group and girls without a peer attachment instability risk were statistically different $(p<0.05)$ in subjective health status, life satisfaction, depression symptom, and self-esteem. The probability of peer attachment instability risk was higher with girls who subjectively recognized unhealthy $(60.0 \%)$, showed depression symptoms $(47.5 \%)$, and had low self-esteem (42.2\%).

\subsection{Back propagation neural network}

The BPN analysis results of learning samples $(63.6 \%)$, testing samples $(27.3 \%)$, and validation samples $(9.1 \%)$ are shown in Figure 1. Five hidden layers generating the least data error were withdrawn. The area under ROC was 0.728 , indicating that the fit and explanatory power of the model satisfied the fair $(0.7-0.8)$ condition (Figure 2).

A BNP model was constructed by selecting the frequency of parent-child dialogue, subjective health status, academic achievement satisfaction, subjective household economy level, depression symptom, self-esteem, subjective life satisfaction, and mobile phone dependency as input variables (Figure 3).

The importance of independent variables in the BPN model was analyzed (Figure. 4). Depression symptom (0.373), self-esteem (0.207), parent-child dialogue level (0.128), and subjective health status $(0.15)$ were withdrawn as the major variables for predicting peer attachment instability, which had higher prediction weight. The normalized importance, which uniformed the units of input variable importance and the influence level of input variables, was shown in Table 2. The most important predictor of peer attachment instability was depression, followed by self-esteem, parent-child dialogue level, and subjective health status in the order of magnitude. 
Table 1: Characteristics of the Subjects Based on Peer Attachment, N (Percentage)

\begin{tabular}{|c|c|c|c|}
\hline Variables & Insecure pee & & $\mathrm{p}$ \\
\hline & No $(n=161)$ & Yes $(n=48)$ & \\
\hline Subjective health status & & & 0.004 \\
\hline Healthy & $157(78.9)$ & $42(21.1)$ & \\
\hline Satisfaction of academic achievement & & & 0.791 \\
\hline Satisfaction & $84(77.8)$ & $24(22.2)$ & \\
\hline Not satisfied & $77(76.2)$ & $24(23.8)$ & \\
\hline Satisfaction of Life & & & 0.003 \\
\hline Not satisfied & $10(50.0)$ & $10(50.0)$ & \\
\hline Subjective level of household economy & & & 0.913 \\
\hline Poverty & $96(76.2)$ & $30(23.8)$ & \\
\hline Normal & $59(78.7)$ & $16(21.3)$ & \\
\hline Wealth & $6(75.0)$ & $2(25.0)$ & \\
\hline Dependency of cell phone & & & 0.694 \\
\hline Non-dependent & $117(78.0)$ & $33(22.0)$ & \\
\hline Depressive symptoms & & & $<0.001$ \\
\hline Non-depressed & $130(86.7)$ & $20(13.3)$ & \\
\hline Depression (lower 25\%) & $31(52.5)$ & $28(47.5)$ & \\
\hline Self-esteem & & & 0.001 \\
\hline Normal & $135(82.3)$ & $29(17.7)$ & \\
\hline Low (lower $25 \%$ ) & $26(57.8)$ & $19(42.2)$ & \\
\hline Parent-child dialogue frequency about school life & & & 0.122 \\
\hline Less than twice a week & $88(72.7)$ & $33(27.3)$ & \\
\hline 3-4 times a week & $36(78.3)$ & $10(21.7)$ & \\
\hline More than 5 times a week & $37(88.1)$ & $5(11.9)$ & \\
\hline
\end{tabular}

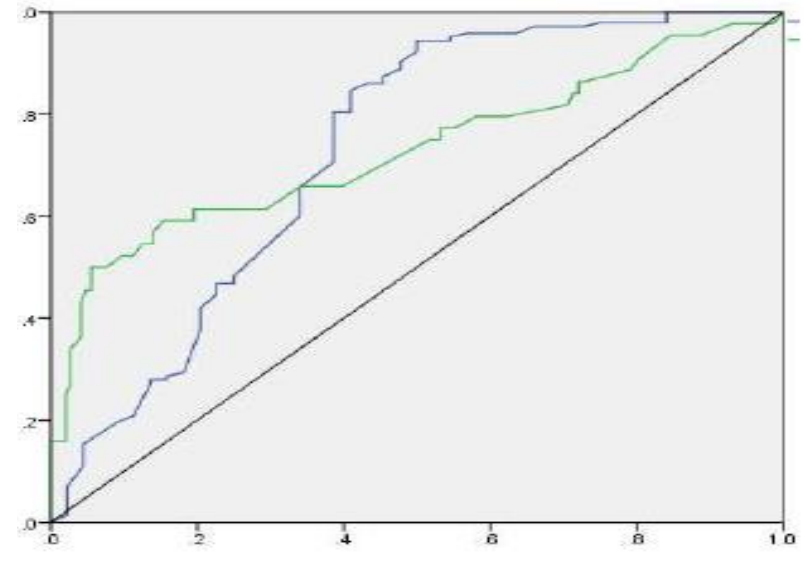

Fig. 2: ROC Curve of Neural Network Algorithm.

Table 2: Relative Importance of Inputs

\begin{tabular}{lll}
\multicolumn{3}{c}{ Table 2: Relative Importance of Inputs } \\
\hline Inputs & $\begin{array}{l}\text { Relative im- } \\
\text { portance }\end{array}$ & $\begin{array}{l}\text { Normalized im- } \\
\text { portance (\%) }\end{array}$ \\
\hline $\begin{array}{l}\text { Subjective health status } \\
\text { Satisfaction of academic }\end{array}$ & 0.115 & 30.9 \\
achievement & 0.042 & 11.4 \\
Satisfaction of Life & 0.053 & 14.2 \\
Subjective level of household & 0.053 & 14.2 \\
economy & 0.029 & 7.9 \\
$\begin{array}{l}\text { Dependency of cell phone } \\
\text { Depressive symptoms }\end{array}$ & 0.373 & 100 \\
Self-esteem & 0.207 & 55.6 \\
$\begin{array}{l}\text { Parent-child dialogue frequency } \\
\text { about school life }\end{array}$ & 0.128 & 34.3 \\
\hline
\end{tabular}

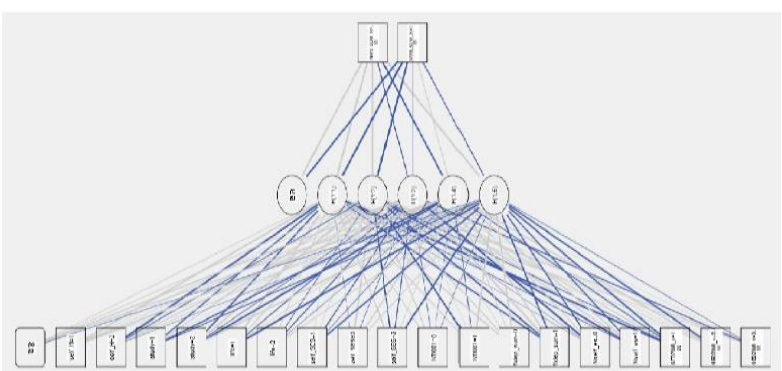

Fig. 3: Synaptic Weighted Network Diagram.

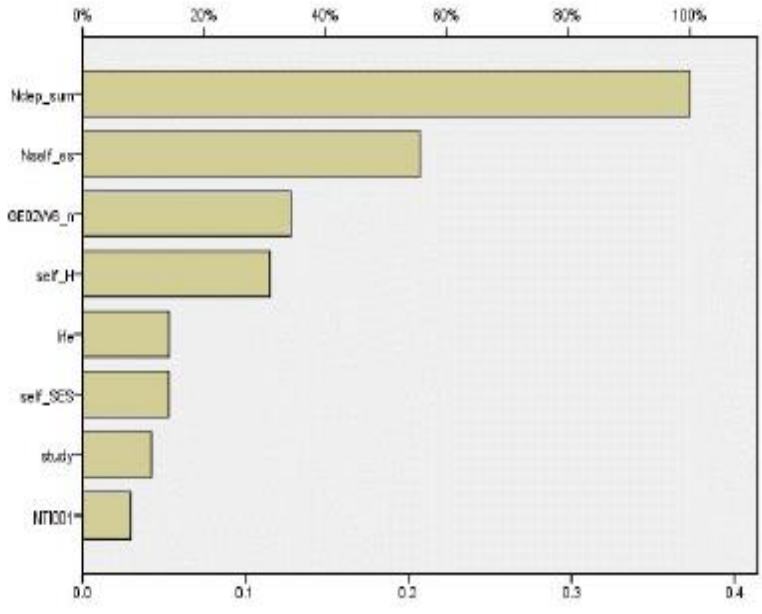

Fig. 4: Normalized Importance.

\section{Discussion}

Factors affecting the peer attachment of South Korean female middle school students were explored by using the BPN algorithm. The results showed that depression, self-esteem, parentchild dialogue level, and subjective health status were main factors affecting the formation of peer attachment formation.

Previous studies evaluating the factors related to peer attachment showed that peer attachment was closely related to personal factors, family factors, and school factors [2], [15]. In these studies, personal factors were self-esteem, stress, life satisfaction, depression, and self-efficacy, school factor was academic achievement, and family factors were socioeconomic status and family conflict [2], [16]. Particularly, the acknowledgment and approval of a parent affected the peer attachment formation of female students 17. Moreover, a previous study reported that the close and positive relationship with parents positively affected the peer attachment formation [9]. These results supported the results of this study, which predicted the parent-child dialogue level as a major variable determining the peer attachment.

This study found that personal health factors such as depression were major factors affecting peer attachment. Particularly, depres- 
sion symptoms were the top priority factor of peer attachment. It was known that unstable peer attachment relationship and depression had a close relationship 10,18 . It was believed that depression symptom was the major predictor of peer attachment because interpersonal behavior associated with depression covariated with depression symptoms or SOMETHING affected interpersonal relationship through the interaction with stress [19], [20]. Therefore, it will be necessary to give an attention and counseling opportunity to depressed adolescents preferentially for successful peer attachment during the adolescence period.

\section{Conclusion}

In summary, female students with low self-esteem, low dialogue level with a parent, a depression symptom, and subjectively perceived unhealthy have a high risk of peer attachment instability. Therefore, we have to actively seek a way to identify the attachment level of the high-risk group and help them adapt to life with peers.

It is required to develop a customized peer attachment enhancement program considering the family factors as well as personal characteristics based on the results of this study.

\section{References}

[1] Arnett J J, Adolescence and emerging adulthood, Pearson, Boston, MA, 2014

[2] Lee H, Jung E, A study on prediction models for adolescent attachment types of parents, teachers and peers using data mining, The Korean Journal Child Education, 2016, 25, pp. 23-38.

[3] Rueger SY, Malecki CK, Demaray M K, Relationship between multiple sources of perceived social support and psychological and academic adjustment in early adolescence: Comparisons across gender, Journal of Youth and Adolescence, 2010, 39, pp. 47.

[4] Armsden GC, Greenberg M T, The inventory of parent and peer attachment: Individual differences and their relationship to psychological well-being in adolescence, Journal of youth and adolescence, 1987, 16, pp. 427-54.

[5] Min WS, Park WM, Cheon SM, Lee Y S, The relationships among social anxiety, aggression and peer relations affected by adult attachment types for the 5 th and 6 th graders in elementary school, Journal of Emotional \& Behavioral Disorders, 2007, 23, pp. 11540.

[6] Pallini S, Baiocco R, Schneider B H, Madigan S, Atkinson L, Early child-parent attachment and peer relations: A meta-analysis of recent research, Journal of Family Psychology, 2014, 28, pp. 118-23.

[7] Buist K L, Deković M, Prinzie P, Sibling relationship quality and psychopathology of children and adolescents: A meta-analysis, Clinical Psychology Review, 2013, 33, pp. 97-106.

[8] Ma CQ, Huebner ES, Attachment relationships and adolescents' life satisfaction: Some relationships matter more to girls than boys, Psychology in the Schools, 2008; 45, pp. 177-90.

[9] Gorrese A, Ruggieri R, Peer attachment: A meta-analytic review of gender and age differences and associations with parent attachment, Journal of youth and adolescence, 2002, 41, pp. 650-72.

[10] Kim JC, Gyeong JS, Choe WH, Effects of parents, teachers and friends` attachment on aggression in early adolescence, The Korean Journal Child Education, 2010, 19, pp. 97-113.

[11] Pradhan B, Lee S, Buchroithner M F, A GIS-based backpropagation neural network model and its cross application and validation for landslide susceptibility analyses. Computers, Environment and Urban Systems, 2010, 34, pp. 216-35.

[12] Korea Youth Policy Institute, Panel survey of local children's centers, Korea Youth Policy Institute, Seoul, 2015.

[13] Hwang M K, The relationship between parent-peer attachment of multi-culture children and social anxiety, Pukyong University, Med. thesis, Korea, 2010.

[14] Byeon H, Cho S, The Factors of Subjective Voice Disorder Using Integrated Method of Decision Tree and Multi-Layer Perceptron Artificial Neural Network Algorithm, International Journal of Advanced Computer Science and Applications, 2016; 7, pp. 112-16.

[15] Ko Y, The relationship among father, mother, peer attachment and subjective well-being in middle school students, The Journal of Yeolin Education, 2008, 16, pp. 111-31.
[16] Meeus WI M, Oosterwegel A, Vollebergh W, Parental and peer attachment and identity development in adolescence, Journal of adolescence, 2002, 25, pp. 93-106.

[17] No B O, Park S, Yi S, Park H J, Trajectories of adolescents' peer attachment and their predictors: A multiple group analysis according to gender,Studies on Korean Youth, 2016, 27, pp. 149-77.

[18] Millings A, Buck R, Montgomery A, Spears M, Stallard P, School connectedness, peer attachment, and self-esteem as predictors of adolescent depression, Journal of adolescence, 2012, 35, pp. 106167.

[19] Kwon H I, Ham B J, Paik JW, Suh SY, Kwon J H, Psychosocial functioning in depression, Korean Journal of Clinical Psychology, 2010, 29, pp. 1117-33.

[20] Nelis S M, Rae G, Brief report: Peer attachment in adolescents, Journal of adolescence, 2009, 32, pp. 443-7. 